\title{
Appalachian youth e-cigarette harm perceptions and tobacco use
}

\author{
Delvon T. Mattingly ${ }^{1}$, Joy L. Hart ${ }^{1,2,3}$, Thanh-Huyen T. Vu ${ }^{3,4}$, Kandi L. Walker ${ }^{1,2,3}$
}

\author{
AFFILIATION \\ 1 Department of Communication, University of Louisville, Louisville, \\ United States \\ 2 Christina Lee Brown Envirome Institute, Louisville, United States \\ 3 American Heart Association Tobacco Center for Regulatory Science, \\ Dallas, United States \\ 4. Department of Preventive Medicine, Feinberg School of Medicine, \\ Northwestern University, Chicago, United States
}

CORRESPONDENCE TO

Joy L. Hart. Department of Communication, University of Louisville, 2301S 3rd St, Louisville, KY 40292, United States. E-mail: joy.hart@louisville.edu

\section{KEYWORDS}

tobacco, youth, e-cigarettes, Appalachia, harm perceptions

Received: 5 August 2020, Revised: 2 January 2021, Accepted: 3 January 2021

https://doi.org/10.18332/popmed/132120 problems (18.7\%) and that e-cigarettes are addictive (25.1\%), compared to never and non-e-cigarette users. More e-cigarette users strongly agreed (32.3\%) that e-cigarettes are healthier alternatives to cigarettes compared to never $(24.1 \%)$ and non-e-cigarette $(25.7 \%)$ users. Regression models indicate that e-cigarette users had greater odds of strongly disagreeing with e-cigarettes being harmful or addictive, compared to never users. E-cigarette users had approximately six-fold odds of strongly disagreeing with e-cigarettes causing health problems $(\mathrm{OR}=6.12 ; 95 \% \mathrm{CI}$ : 3.16-11.90) and two times greater odds of disagreeing with e-cigarettes being addictive (OR=2.09; 95\% CI: $1.07-4.08$ ) compared to never users.

CONCLUSIONS Appalachian youth ever e-cigarette users perceive e-cigarettes as less harmful and less addictive compared to never users. Better informing youth, especially youth tobacco users, of the harms associated with e-cigarettes is vital.

\section{INTRODUCTION}

For at least the past 5 years, e-cigarettes have been the most commonly used tobacco product among youth ${ }^{1}$. In 2019, more than a third of US middle and high students had ever used e-cigarettes and one fifth were current users ${ }^{2}$. Although e-cigarettes have been marketed as healthier alternatives to conventional cigarettes, their long-term health effects are not well documented, especially for youth users, and there is no consensus regarding their utility for smoking cessation $^{3}$. Preventing youth tobacco uptake and established use are public health concerns that tend to garner more attention than promoting cessation, and e-cigarette use rates demonstrate their appeal to this population ${ }^{4-7}$. Because e-cigarettes normally contain nicotine, youth users are at risk for the harms associated with nicotine consumption, such as epigenetic changes to the brain making them prone to future substance use $\mathrm{e}^{8}$. Thus, better understanding factors that influence e-cigarette use in youth is important in preventing future use and its associated health implications, especially for youth vulnerable to tobacco use.

Appalachian youth are disproportionately exposed to tobacco. The Appalachian region has a history of tobacco culture from tobacco production to acceptance of tobacco use and celebrating this heritage (e.g. tobacco festivals) ${ }^{9}$. As a result, youth in Appalachia have higher smoking prevalence than youth residing in other parts of the US ${ }^{10}$. In addition to culture, rurality and poverty are associated with increased tobacco use ${ }^{11,12}$, possibly contributing to higher rates of use 
among Appalachia youth. Accordingly, Appalachian youth are at risk for tobacco use, including the use of e-cigarettes.

Studies have linked youth e-cigarette use to viewing e-cigarettes as less harmful or less addictive than combustible cigarettes ${ }^{5-7}$. Additional evidence suggests that youth perceive e-cigarettes as less harmful than other tobacco products, such as cigars and smokeless tobacco ${ }^{13}$. Although a relationship between perceiving e-cigarettes as less harmful or less addictive and e-cigarette use has been documented with some youth samples ${ }^{5-7}$, research on Appalachian youth is limited. One study found that Appalachian youth perceived e-cigarettes as causing fewer health problems and less addiction than conventional tobacco products ${ }^{14}$. Our study extends prior research by examining specific e-cigarette-related harms, such as whether e-cigarettes cause breathing and oral health problems, as well as whether these perceptions differ by tobacco use.

\section{METHODS}

\section{Study design}

The Youth Appalachian Tobacco Study (n=1074), a crosssectional survey of tobacco exposures, use patterns, and perceptions and attitudes, was conducted from 2014-2016. The Appalachian states Kentucky, North Carolina, and New York, were selected based on overall tobacco use rates (high, medium, and low, respectively). High school and middle school youth from Appalachian counties in each state were sampled, and participants completed a questionnaire during a regular school day. Detailed information on sampling and participants has been published elsewhere ${ }^{14}$. The study was approved by the University of Louisville's Institutional Review Board.

\section{Measures}

E-cigarette harm perceptions

In the Youth Appalachian Tobacco Study, youth were asked to indicate whether they strongly disagree, disagree, neither agree nor disagree, agree, or strongly agree (using 1-5 rating scales) with the following six statements indicating that use of e-cigarettes: 1) causes health problems, 2) is healthier than smoking traditional cigarettes, 3) causes breathing problems, 4) causes oral health problems, 5) is addictive, and 6) is difficult to quit.

\section{Tobacco use}

Tobacco use was categorized into three groups: never users, ever non-e-cigarette users (i.e. cigarettes, smokeless tobacco), and ever e-cigarette users. Never users were participants who had never used cigarettes, e-cigarettes, or smokeless tobacco. Ever non-e-cigarette users were participants who had ever used cigarettes or smokeless tobacco but not e-cigarettes (referred to below as non-ecigarette users). Ever e-cigarette users were participants who had ever used e-cigarettes (referred to below as e-cigarette users). The distribution of tobacco use is presented in Supplementary file Table S1.

\section{Covariates}

Gender (male, female), race/ethnicity (White/Caucasian, nonWhite/non-Caucasian), age, school type (middle, high), state (New York, Kentucky, North Carolina), and number of household tobacco users (zero, $\geq 1$ ) were included in this analysis.

\section{Statistical analysis}

The distribution of each e-cigarette harm perception was calculated overall and by tobacco use. Pairwise comparisons were conducted between: 1) never users and e-cigarette users, 2) never users and non-e-cigarette users, and 3) non-e-cigarette users and e-cigarette users. Multivariable multinomial logistic regression models were used to estimate odds ratio (OR) and 95\% confidence interval (CI) for associations between each harm perception as a categorical variable (Reference: strongly agree) and e-cigarette use (Reference: never users), adjusted for gender, race/ethnicity, school type, state, and household tobacco users. Because age may modify the association between harm perceptions and tobacco use, analyses were performed for the whole sample, followed by a sensitivity analysis excluding youth aged 11-12 years. Analyses were conducted in Stata 16.1.

\section{RESULTS}

About two-thirds (66.1\%) of participants were never users, one-tenth $(10.5 \%)$ were non-e-cigarette users, and onefourth $(23.4 \%)$ were e-cigarette users. The distribution of descriptive characteristics by tobacco use is provided in Supplementary file Table S2. More non-e-cigarette users were female $(60.2 \%)$, and more e-cigarette users were male $(53.4 \%)$. Both use groups were older than never users, and frequencies of non-e-cigarette and e-cigarette use varied by state.

Prevalence of e-cigarette harm perceptions by tobacco use is presented in Table 1. Never users strongly agreed at higher proportions that e-cigarettes cause health problems (34.5\%), breathing problems $(34.1 \%)$, and oral health problems (32.7\%) as well as are addictive (37.3\%) and difficult to quit (34.1\%), compared to non-e-cigarette users and e-cigarette users. Non-e-cigarette users strongly agreed (25.7\%) and agreed $(29.2 \%)$ at higher proportions than never users that e-cigarettes are healthier than smoking traditional cigarettes; however, e-cigarette users most often strongly agreed $(32.3 \%)$. Compared to never and non-e-cigarette users, a higher proportion of e-cigarette users strongly disagreed that e-cigarettes cause health problems (10.4\%), breathing problems $(9.6 \%)$, and oral health problems $(11.6 \%)$ and are difficult to quit $(12.0 \%)$. The distribution of harm perceptions differed between never users and e-cigarette users $(p<0.05)$. However, when comparing never users to non-e-cigarette users, harm perceptions differed only for e-cigarettes cause health problems $(\mathrm{p}=0.020)$ and 
Table 1. Prevalence of e-cigarette harm perceptions by tobacco use $(\mathrm{N}=1074)$

\begin{tabular}{|c|c|c|c|c|c|}
\hline Harm perceptions & $\begin{array}{l}\text { Total } \\
\text { n (\%) }\end{array}$ & $\begin{array}{c}\text { Never users } \\
\text { n (\%) }\end{array}$ & $\begin{array}{c}\text { Ever non-e- } \\
\text { cigarette users* } \\
\text { n (\%) }\end{array}$ & $\begin{array}{c}\text { Ever e-cigarette } \\
\text { users } \\
\text { n (\%) }\end{array}$ & $\mathbf{p}$ \\
\hline Population & $1074(100)$ & $710(66.1)$ & $113(10.5)$ & $251(23.4)$ & \\
\hline Causes health problems & & & & & $<0.001^{\mathrm{a}}$ \\
\hline Strongly disagree & $65(6.1)$ & $31(4.4)$ & $8(7.1)$ & $26(10.4)$ & $0.020^{b}$ \\
\hline Disagree & $104(9.7)$ & $48(6.8)$ & $15(13.3)$ & $41(16.3)$ & $0.12^{\mathrm{c}}$ \\
\hline Neither & $314(29.2)$ & $192(27.0)$ & $37(32.7)$ & $85(33.9)$ & \\
\hline Agree & $278(25.9)$ & $194(27.3)$ & $23(20.4)$ & $61(24.3)$ & \\
\hline Strongly agree & $313(29.1)$ & $245(34.5)$ & $30(26.6)$ & $38(15.1)$ & \\
\hline $\begin{array}{l}\text { Is healthier than smoking traditional } \\
\text { cigarettes }\end{array}$ & & & & & $0.020^{\mathrm{a}}$ \\
\hline Strongly disagree & $105(9.8)$ & $74(10.4)$ & $12(10.6)$ & $19(7.6)$ & $0.78^{\mathrm{b}}$ \\
\hline Disagree & $107(10.0)$ & $67(9.4)$ & $12(10.6)$ & $28(11.2)$ & $0.69^{c}$ \\
\hline Neither & $293(27.3)$ & $212(29.9)$ & $27(23.9)$ & $54(21.5)$ & \\
\hline Agree & $288(26.8)$ & $186(26.2)$ & $33(29.2)$ & $69(27.5)$ & \\
\hline Strongly agree & $281(26.2)$ & $171(24.1)$ & $29(25.7)$ & $81(32.3)$ & \\
\hline Causes breathing problems & & & & & $<0.001^{\mathrm{a}}$ \\
\hline Strongly disagree & $57(5.3)$ & $26(3.7)$ & $7(6.2)$ & $24(9.6)$ & $0.34^{\mathrm{b}}$ \\
\hline Disagree & $87(8.1)$ & $45(6.3)$ & $11(9.7)$ & $31(12.4)$ & $0.35^{c}$ \\
\hline Neither & $309(28.8)$ & $196(27.6)$ & $31(27.4)$ & $82(32.7)$ & \\
\hline Agree & $297(27.7)$ & $201(28.3)$ & $33(29.2)$ & $63(25.1)$ & \\
\hline Strongly agree & $324(30.2)$ & $242(34.1)$ & $31(27.4)$ & $51(20.3)$ & \\
\hline Causes oral health problems & & & & & $<0.001^{\mathrm{a}}$ \\
\hline Strongly disagree & $65(6.1)$ & $27(3.8)$ & $9(8.0)$ & $29(11.6)$ & $0.15^{b}$ \\
\hline Disagree & $99(9.2)$ & $53(7.5)$ & $10(8.9)$ & $36(14.3)$ & $0.13^{c}$ \\
\hline Neither & $350(32.6)$ & 219 (30.9) & 37 (32.7) & $94(37.5)$ & \\
\hline Agree & $254(23.7)$ & $179(25.2)$ & $30(26.6)$ & $45(17.9)$ & \\
\hline Strongly agree & $306(28.5)$ & $232(32.7)$ & 27 (23.9) & 47 (18.7) & \\
\hline Is addictive & & & & & $<0.001^{\mathrm{a}}$ \\
\hline Strongly disagree & $60(5.6)$ & $33(4.7)$ & $9(8.0)$ & $18(7.2)$ & $0.09^{b}$ \\
\hline Disagree & $86(8.0)$ & $46(6.5)$ & $12(10.6)$ & $28(11.2)$ & $0.89^{c}$ \\
\hline Neither & $268(25.0)$ & $162(22.8)$ & $29(25.7)$ & $77(30.7)$ & \\
\hline Agree & 302 (28.1) & 204 (28.7) & $33(29.2)$ & $65(25.9)$ & \\
\hline Strongly agree & 358 (33.3) & 265 (37.3) & $30(26.6)$ & $63(25.1)$ & \\
\hline Is difficult to quit & & & & & $<0.001^{\mathrm{a}}$ \\
\hline Strongly disagree & $77(7.2)$ & $36(5.1)$ & $11(9.7)$ & $30(12.0)$ & $0.002^{b}$ \\
\hline Disagree & $105(9.8)$ & $51(7.2)$ & $17(15.0)$ & 37 (14.7) & $0.97^{c}$ \\
\hline Neither & 315 (29.3) & $209(29.4)$ & $35(31.0)$ & $71(28.3)$ & \\
\hline Agree & $259(24.1)$ & $172(24.2)$ & 27 (23.9) & $60(23.9)$ & \\
\hline Strongly agree & $318(29.6)$ & $242(34.1)$ & $23(20.4)$ & $53(21.1)$ & \\
\hline
\end{tabular}

*Ever non-e-cigarette users include cigarette and smokeless tobacco users who have not tried e-cigarettes. a Chi-squared test comparing never users to ever e-cigarette users. b Chi-squared test comparing never users to ever non-e-cigarette users. c Chi-squared test comparing ever non-e-cigarette users to ever e-cigarette users. 
are difficult to quit ( $\mathrm{p}=0.002)$. Comparisons between non-ecigarette users and e-cigarettes users were not statistically significant. Additionally, sensitivity analysis results suggest that age did not play a significant role in associations between harm perceptions and e-cigarette use.

Results from regression analyses can be found in Table 2 , and values of OR with $95 \%$ CI are reported below. Compared to never users, e-cigarette users had greater odds of strongly disagreeing (OR=6.12; 95\% CI: 3.16-11.90) and disagreeing (OR=4.78; 95\% CI: 2.71-8.41) that e-cigarettes cause health problems. Also, compared to never users, non-e-cigarette users had greater odds of disagreeing $(\mathrm{OR}=2.30 ; 95 \% \mathrm{CI}: 1.11-4.75)$ that e-cigarettes cause health problems. E-cigarette users also had more than fourfold odds of strongly disagreeing (OR=4.40; 95\% CI: 2.26-8.56) and over threefold odds of disagreeing $(\mathrm{OR}=3.14 ; 95 \% \mathrm{CI}$ : 1.76-5.60) that e-cigarettes cause breathing problems, compared to never users; no associations were found for non-e-cigarette users. Similarly, e-cigarette users (OR=6.01; 95\% CI: 3.16-11.44) and non-e-cigarette users (OR=3.34; 95\% CI: 1.37-8.15) had greater odds of strongly disagreeing that e-cigarettes cause oral health problems, compared to never users. Both e-cigarette and non-e-cigarette users had elevated odds of strongly disagreeing or disagreeing that e-cigarettes are addictive and difficult to quit. Associations for e-cigarettes being difficult to quit were similar with both groups having at least threefold odds of strongly disagreeing or disagreeing compared to never users.

Table 2. Multivariable multinomial logistic regression modeling associations between harm perceptions and ever non-e-cigarette use and harm perceptions and ever e-cigarette use $(\mathrm{N}=1074)$

\begin{tabular}{|c|c|c|}
\hline Harm perceptions & $\begin{array}{l}\text { Ever non-e- } \\
\text { cigarette use* } \\
\text { vs never use } \\
\text { OR }(95 \% \mathrm{CI})\end{array}$ & $\begin{array}{c}\text { Ever e-cigarette } \\
\text { use vs never } \\
\text { use } \\
\text { OR }(95 \% \mathrm{CI})\end{array}$ \\
\hline \multicolumn{3}{|c|}{ Causes health problems } \\
\hline Strongly disagree & $2.39(0.97-5.92)$ & $6.12(3.16-11.90)$ \\
\hline Disagree & $2.30(1.11-4.75)$ & $4.78(2.71-8.41)$ \\
\hline Neither & $1.63(0.95-2.80)$ & $2.72(1.74-4.25)$ \\
\hline Agree & $0.95(0.52-1.72)$ & $1.86(1.17-2.97)$ \\
\hline Strongly agree & Ref. & Ref. \\
\hline \multicolumn{3}{|l|}{$\begin{array}{l}\text { Is healthier than } \\
\text { smoking traditional } \\
\text { cigarettes }\end{array}$} \\
\hline Strongly disagree & $0.99(0.47-2.12)$ & $0.58(0.32-1.05)$ \\
\hline Disagree & $0.96(0.45-2.05)$ & $0.86(0.50-1.49)$ \\
\hline Neither & $0.79(0.44-1.41)$ & $0.54(0.36-0.82)$ \\
\hline Agree & $1.04(0.59-1.83)$ & $0.74(0.50-1.11)$ \\
\hline Strongly agree & Ref. & Ref. \\
\hline
\end{tabular}

Table 2. Continued

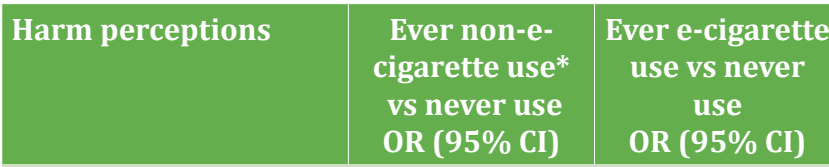

\section{Causes breathing} problems

$\begin{array}{lll}\text { Strongly disagree } & 2.13(0.82-5.50) & \mathbf{4 . 4 0}(\mathbf{2 . 2 6 - 8 . 5 6}) \\ \text { Disagree } & 1.94(0.88-4.28) & \mathbf{3 . 1 4}(\mathbf{1 . 7 6 - 5 . 6 0}) \\ \text { Neither } & 1.21(0.69-2.11) & \mathbf{1 . 8 5}(\mathbf{1 . 2 2 - 2 . 8 1}) \\ \text { Agree } & 1.24(0.72-2.15) & 1.34(0.87-2.06)\end{array}$

Strongly agree

Ref.

Ref.

\section{Causes oral health problems}

\begin{tabular}{|c|c|c|}
\hline Strongly disagree & $3.34(1.37-8.15)$ & $6.01(3.16-11.44)$ \\
\hline Disagree & $1.69(0.75-3.82)$ & $3.15(1.80-5.51)$ \\
\hline Neither & $1.51(0.87-2.62)$ & $2.00(1.32-3.03)$ \\
\hline Agree & $1.41(0.79-2.52)$ & $1.1(0.69-1.78)$ \\
\hline Strongly agree & Ref. & Ref. \\
\hline \multicolumn{3}{|l|}{ Is addictive } \\
\hline Strongly disagree & $2.26(0.95-5.42)$ & $2.09(1.07-4.08)$ \\
\hline Disagree & $2.31(1.07-5.02)$ & $2.31(1.30-4.09)$ \\
\hline Neither & $1.34(0.75-2.37)$ & $1.67(1.11-2.50)$ \\
\hline Agree & $1.30(0.75-2.25)$ & $1.14(0.76-1.72)$ \\
\hline Strongly agree & Ref. & Ref. \\
\hline \multicolumn{3}{|l|}{ Is difficult to quit } \\
\hline Strongly disagree & $3.21(1.39-7.41)$ & $3.61(1.98-6.58)$ \\
\hline Disagree & $3.77(1.82-7.82)$ & $3.21(1.86-5.54)$ \\
\hline Neither & $1.52(0.85-2.72)$ & $1.27(0.83-1.93)$ \\
\hline Agree & $1.61(0.88-2.97)$ & $1.45(0.94-2.25)$ \\
\hline Strongly agree & Ref. & Ref. \\
\hline
\end{tabular}

*Ever non-e-cigarette users include cigarette and smokeless tobacco users who have not tried e-cigarettes. Models adjusted for gender, race/ethnicity, school type, state, and number of household tobacco users. Ref.: reference group.

\section{DISCUSSION}

Compared to never users, Appalachian youth e-cigarette users and non-e-cigarette users perceive e-cigarettes to be less harmful, less addictive and less difficult to quit, with harm-related associations stronger for e-cigarette users. E-cigarette users disagreed that problems with breathing and oral health were caused by e-cigarette use, despite recent evidence of a potential link between e-cigarette use and respiratory disease ${ }^{15}$. Associations were less clear for non-e-cigarette users, illustrating ways that perceptions vary by type of health issue and use pattern. Among e-cigarette 
users, associations appear to be stronger for health-related harms, and addiction-related harms were similar among none-cigarette and e-cigarette users.

Our findings corroborate previous research that reports associations between perceiving e-cigarettes as less harmful or less addictive and tobacco use among youth ${ }^{5,6}$. Specifically, among youth never cigarette users, perceiving e-cigarettes as less harmful was associated with e-cigarette use ${ }^{5}$. Similarly, among a sample of Florida youth, perceiving e-cigarettes as easy to quit was associated with more e-cigarette use including concurrent use of e-cigarettes and other tobacco products 6 . In our study, Appalachian youth views on whether e-cigarettes are harmful or addictive varied by tobacco use patterns. Although e-cigarette users indicated less concern about harms than did non-e-cigarette users, results for the latter group, who already use tobacco, suggest risk for e-cigarette uptake.

To combat e-cigarette use, health campaigns must inform youth of the associated harms and potential for addiction. Given evidence that perceiving e-cigarettes as less harmful predicts subsequent use among never users ${ }^{7}$, efforts must better explicate factors that predict youth susceptibility to trying e-cigarettes. Further, targeted messaging to e-cigarette users, who tend to perceive e-cigarettes as less dangerous, is needed to raise awareness and increase understanding, as these youth are especially vulnerable to continued use.

\section{Limitations}

Our study has limitations. First, responses were selfreported and thus are susceptible to associated biases. Second, the study is cross-sectional; thus, directionality cannot be determined. Third, the study was conducted prior to the COVID-19 pandemic, which may have altered harm perceptions related to tobacco use. Despite these limitations, our study is among the first to observe associations between perceiving e-cigarettes as less harmful or less addictive and e-cigarette use in a sample of Appalachian youth.

\section{CONCLUSIONS}

Compared to never users, Appalachian youth non-ecigarette and e-cigarette users perceive e-cigarettes as less harmful, with associations greater in magnitude for e-cigarette users. These users disagree that e-cigarettes are harmful, cause oral health problems, are addictive, and are difficult to quit. Our findings, which support previous work indicating that perceiving e-cigarettes as less harmful or less addictive is associated with e-cigarette use, provide insights on Appalachian youth tobacco views and use. Health communication messaging should address increasing youth understanding of the health dangers of these products, especially for vulnerable youth.

\section{REFERENCES}

1. Gentzke AS, Creamer M, Cullen KA, et al. Vital Signs: Tobacco Product Use Among Middle and High School Students -
United States, 2011-2018. MMWR Morb Mortal Wkly Rep. 2019;68(6):157-164. doi:10.15585/mmwr.mm6806e1

2. Wang TW, Gentzke AS, Creamer MR, et al. Tobacco Product Use and Associated Factors Among Middle and High School Students - United States, 2019. MMWR Surveill Summ. 2019;68(12):1-22. doi:10.15585/mmwr.ss6812a1

3. Drummond MB, Upson D. Electronic cigarettes. Potential harms and benefits. Ann Am Thorac Soc. 2014;11(2):236242. doi:10.1513/AnnalsATS.201311-391FR

4. Padon AA, Lochbuehler K, Maloney EK, Cappella JN. A Randomized Trial of the Effect of Youth Appealing E-Cigarette Advertising on Susceptibility to Use E-Cigarettes Among Youth. Nicotine Tob Res. 2018;20(8):954-961. doi:10.1093/ ntr/ntx155

5. Amrock SM, Zakhar J, Zhou S, Weitzman M. Perception of e-cigarette harm and its correlation with use among U.S. adolescents. Nicotine Tob Res. 2015;17(3):330-336. doi:10.1093/ntr/ntu156

6. Bernat D, Gasquet N, Wilson KO, Porter L, Choi K. Electronic Cigarette Harm and Benefit Perceptions and Use Among Youth. Am J Prev Med. 2018;55(3):361-367. doi:10.1016/j. amepre.2018.04.043

7. Brose LS, Brown J, Hitchman SC, McNeill A. Perceived relative harm of electronic cigarettes over time and impact on subsequent use. A survey with 1-year and 2-year followups. Drug Alcohol Depend. 2015;157:106-111. doi:10.1016/j. drugalcdep.2015.10.014

8. Yuan M, Cross SJ, Loughlin SE, Leslie FM. Nicotine and the adolescent brain. J Physiol. 2015;593(16):3397-3412. doi:10.1113/JP270492

9. Hart JL, Walker KL, Sears CG, et al. The 'state' of tobacco: Perceptions of tobacco among Appalachian youth in Kentucky. Tob Prev Cessation. 2018;4(January):1-5. doi:10.18332/tpc/81857

10. Centers for Disease Control and Prevention. Map of Current Cigarette Use Among Youth: Current Cigarette Use Among Youth (Youth Risk Behavior Surveillance System) 2017*. https://www.cdc.gov/statesystem/cigaretteuseyouth.html. Updated September 14, 2020. Accessed June 2020.

11. Creamer MR, Wang TW, Babb S, et al. Tobacco product use and cessation indicators among adults-United States, 2018. MMWR Morb Mortal Wkly Rep. 2019;68(45):1013-1019. doi:10.15585/mmwr.mm6845a2

12. Lutfiyya MN, Shah KK, Johnson M, et al. Adolescent daily cigarette smoking: is rural residency a risk factor?. Rural Remote Health. 2008;8(1):875. PMID:18366278.

13. Amrock SM, Lee L, Weitzman M. Perceptions of e-Cigarettes and Noncigarette Tobacco Products Among US Youth. Pediatrics. 2016;138(5):e20154306. doi:10.1542/peds.20154306

14. Mattingly DT, Tompkins LK, Rai J, Sears CG, Walker KL, Hart JL. Tobacco use and harm perceptions among Appalachian youth. Prev Med Rep. 2020;18:101089. doi:10.1016/j. pmedr.2020.101089

15. Wills TA, Soneji SS, Choi K, Jaspers I, Tam EK. E-cigarette 
Use and Respiratory Disorder: An Integrative Review of Converging Evidence from Epidemiological and Laboratory Studies. Eur Respir J. 2020. doi:10.1183/13993003.018152019

\section{ACKNOWLEDGEMENTS}

We acknowledge assistance from Clara Sears, Alex Lee, Courteney Smith, and Allison Siu with data collection and from Shesh Rai with analyses. Also, the resources of the University of Louisville's research computing group and the Cardinal Research Cluster facilitated this research.

\section{CONFLICTS OF INTEREST}

The authors have each completed and submitted an ICMJE form for disclosure of potential conflicts of interest. The authors declare that they have no competing interests, financial or otherwise, related to the current work. J. L. Hart, Thanh-Huyen T. Vu and Kandi L. Walker report grants from the National Institutes of Health during the conduct of the study.

FUNDING

Research reported in this publication was supported, in part, by the
National Heart, Lung, and Blood Institute (NHLBI) of the National Institutes of Health (NIH) and FDA Center for Tobacco Products under Award Numbers P50HL120163 and U54HL120163. The content is solely the responsibility of the authors and does not necessarily represent the official views of the NIH, the Food and Drug Administration, or the American Heart Association. The funding sponsors had no role in study design; data collection, analyses, or interpretation; manuscript preparation; or the decision to publish the results.

PROVENANCE AND PEER REVIEW

Not commissioned; externally peer reviewed. 